Research Article

\title{
Nomogram-Based New Recurrence Predicting System in Early-Stage Papillary Thyroid Cancer
}

\author{
Yongfeng Ding $\mathbb{C D}^{1,2}$ Zhuochao Mao ${ }^{1 D},{ }^{1}$ Jiaying Ruan, ${ }^{1}$ Xingyun Su, ${ }^{1}$ Linrong Li, ${ }^{3}$ \\ Thomas J. Fahey III, ${ }^{4}$ Weibin Wang $\mathbb{D}^{1,2}$ and Lisong Teng $\mathbb{D}^{1,2}$ \\ ${ }^{1}$ Cancer Center, The First Affiliated Hospital, Zhejiang University School of Medicine, Hangzhou, China \\ ${ }^{2}$ Key Laboratory of Precision Diagnosis and Treatment for Hepatobiliary and Pancreatic Tumor of Zhejiang Province, \\ Hangzhou, China \\ ${ }^{3}$ Department of Orthopedics, The Second Affiliated Hospital, Zhejiang University School of Medicine, Hangzhou, China \\ ${ }^{4}$ Department of Surgery, New York Presbyterian Hospital-Weill Cornell Medical College, New York, NY, USA
}

Correspondence should be addressed to Weibin Wang; wbwang@zju.edu.cn and Lisong Teng; lsteng@zju.edu.cn

Yongfeng Ding and Zhuochao Mao contributed equally to this work.

Received 7 April 2019; Revised 31 May 2019; Accepted 3 July 2019; Published 5 September 2019

Academic Editor: Claudio Casella

Copyright (c) 2019 Yongfeng Ding et al. This is an open access article distributed under the Creative Commons Attribution License, which permits unrestricted use, distribution, and reproduction in any medium, provided the original work is properly cited.

\begin{abstract}
Background and Objectives. The clinicopathological risk factors to predict recurrence of papillary thyroid cancer (PTC) patients remain controversial. Methods. PTC patients treated with thyroidectomy between January 1997 and December 2011 at the First Affiliated Hospital of Zhejiang University (Zhejiang cohort) were included. Multivariate Cox regression analysis was conducted to identify independent recurrence predictors. Then, the nomogram model for predicting probability of recurrence was built. Results. According to Zhejiang cohort $(N=1,697)$, we found that the 10 -year event-free survival (EFS) rates of PTC patients with earlystage (TNM stages I, II, and III) were not well discriminated $(91.6 \%, 89.0 \%$, and $90.7 \% ; P=0.768)$. The multivariate Cox model identified age, bilaterality, tumor size, and nodal status as independent risk factors for tumor recurrence in PTC patients with TNM stages I-III. We then developed a nomogram with the C-index 0.70 (95\% CI, 0.64 to 0.76 ), which was significantly higher $(P<0.0001)$ than the AJCC staging system $(0.52)$. In the validation group, the C-index remained at a similar level. Conclusions. In this study, we build up a new recurrence predicting system and establish a nomogram for early-stage PTC patients. This prognostic model may better predict individualized outcomes and conduct personalized treatments.
\end{abstract}

\section{Introduction}

Thyroid cancer is one of the most common human endocrine tumors. In China, South Korea, and other Asian countries, the incidence of thyroid cancer even increased by 3-6 times in recent decades [1-3]. Among all the thyroid cancer, papillary thyroid cancer (PTC) is the most common pathological type, accounting for about $80-85 \%$ patients of total $[4,5]$. The majority of PTC patients are indolent and usually have a favorable prognosis. However, a subgroup of PTC patients (about 5\%) will develop aggressive growth, metastatic spread and loss of response to conventional therapy [6].
The American Joint Committee on Cancer (AJCC) is the most widely used staging system for thyroid cancer to predict prognosis. Meanwhile, this system is mainly focused on the survival rather than the recurrence, and it therefore is not sufficient to predict recurrence especially for those PTC patients of early stages. The American Thyroid Association (ATA) has put forward a revised recurrence risk stratification system for differentiated thyroid cancer (DTC, including PTC patients) in 2015: the modified 2009 ATA Risk Stratification System (M-2009-RSS) [7], in which they redivided patients with DTC into high-, median- and low-risk groups $[8,9]$. So far, since PTC's unique extended survival period, a number of different staging or prognostic scoring 
systems were developed, but the controversy remains, particularly on those low-intermediate risk PTC patients.

A nomogram is a visually predictive tool that provides the probability of specific outcomes, such as overall survival and cancer recurrence, for individual patients [10]. Currently, nomograms have been developed in the majority of cancer types, including thyroid cancers $[11,12]$.

In this study, we found that the EFS of PTC patients with TNM stages I, II, and III was not well discriminated. By using univariate and multivariate Cox regression analysis, we established a new risk stratification system for this specific group of PTCs. A new nomogram was ultimately built to visually predict the probability of recurrence in PTC patients with TNM stages I-III.

\section{Materials and Methods}

2.1. Patients and Study Design. A retrospective study was conducted on a primary cohort of patients who underwent total/near-total thyroidectomy for papillary thyroid cancer between January 1997 and December 2011 at the First Affiliated Hospital, Zhejiang University School of Medicine (Hangzhou, China). Patients who had previous radiation exposure, had a family history of PTC, or underwent previous thyroid surgery were excluded. In addition, patients whose follow-up data were not available were excluded. Meanwhile, by reviewing the medical records and pathology reports, these clinicopathologic characteristics of patients were included as follows: age at diagnosis, sex (male and female), bilaterality (yes and no), tumor size (maximum tumor diameter), extrathyroidal extension (including minimal extrathyroidal extension) (yes and no), and nodal status $\left(\mathrm{N} 0 / \mathrm{Nx}, \mathrm{N} 1 \mathrm{a}\right.$, and $\mathrm{N} 1 \mathrm{~b}$ are defined according to AJCC $7^{\text {th }}$ edition). It should be noted that information related to radioactive iodine (RAI) treatment was not included in the study. This study was approved by the Institutional Review Board of the First Affiliated Hospital, Zhejiang University School of Medicine. Informed consent was obtained before surgery. As we mentioned, all patients were followed postoperatively with measurements of serum thyroglobulin and thyroglobulin antibody, neck ultrasound, and iodine131 whole-body scans to monitor for disease recurrence and survival [13].

2.2. Cox Regression Analysis. Univariate and multivariate Cox regression analyses were conducted to select a subset of independent prognosis predictors for the disease-free survival of PTC. In addition, prognostic index (PI) was calculated using parameters generated by multivariate Cox regression with stepwise selection method.

2.3. Construction and Validation of the Nomogram. To construct the effective clinical nomogram, we randomly divided our patients into a modeling cohort and a validation cohort. First, a nomogram was performed using the training cohort based on the independent prognostic factors identified in multivariate Cox regression analysis. The performance of the nomogram was assessed by discrimination (concordance index, C-index) and calibration (comparing nomogram-predicted versus observed Kaplan-Meier estimates of survival probability) [10]. Internal validation of the nomogram was performed using the modeling cohort. The validation cohort was utilized for external validation.

2.4. Statistical Analysis. Statistical analysis was conducted using SAS 9.4 (SAS Institute, Inc., Cary, NC, USA). Categorical variables were compared using the $\chi^{2}$ test or Fisher's test. The optimal cutoff value was determined using X-tile software [14]. Survival curves were depicted using the Kaplan-Meier method and compared by using the log-rank test. Nomogram analysis was performed using the package of rms [15] in R version 3.3.0 (http://www.r-project.org/). For all of the analyses, $P<0.05$ in a two-tailed test was considered to be statistically significant.

\section{Results}

3.1. Deficiency in TNM Staging System. 1697 patients that met the inclusion criteria were included, and the median follow-up time was 67 months (range of 4 to 230 months). Figure 1 shows that the 10-year EFS rates for TNM I, II, and III were $91.6 \%, 89.0 \%$, and $90.7 \%$, respectively $(P=0.768)$, which were significantly higher than that for PTC patients with TNM IV $(76.5 \%, P<0.0001)$. These results indicated that TNM staging system was not efficient enough to distinguish the EFS rates for PTC patients with TNM stages I-III.

3.2. Clinicopathologic Characteristics of PTC Patients with TNM Stages I-III. 1621 PTC patients with TNM stages I-III from Zhejiang Cohort were included for our further analysis. The median follow-up time was 67 months. The demographics and tumor characteristics of patients are summarized in Table 1. The cohort included 384 (23.7\%) men and 1237 (76.3\%) women with a mean age of $44.1 \pm 11.8$ years. Bilateral tumors were seen in $303(18.7 \%)$ patients. Patients with the maximum tumor diameter $\leq 10 \mathrm{~mm}$ (papillary thyroid microcarcinoma, PTMC) and $>20 \mathrm{~mm}$ accounted for $60.9 \%$ and $11.1 \%$ of the proportion, respectively. The patients with TNM stages I, II, and III were $1420(87.6 \%), 37(2.3 \%)$, and $164(10.1 \%)$, respectively.

3.3. Cutoff Age for Recurrence Prediction. To select the best cutoff value of age to predict disease recurrence in PTC patients, the X-tile software was used. The age of 30 was identified as the best cutoff ( $P$ adjust $=0.001$, Figure $2(\mathrm{a})$ ). Figure 2(b) illustrates the survival curves of EFS for younger than 30 group (age at diagnosis $<30$ ) and elder than 30 group (age at diagnosis $\geq 30) \quad(P=0.001 ; \quad \mathrm{HR}=2.68 ; 95 \%$ $\mathrm{CI}=1.72-4.20)$. Patients who were younger than 30 had a much higher recurrent rate. Thus, we picked age of 30 as cutoff value in our later analysis.

To further assess whether the patients younger than 30 had higher recurrent rate due to lymph node recurrences, we divided the recurrent events into two groups: lymph node 


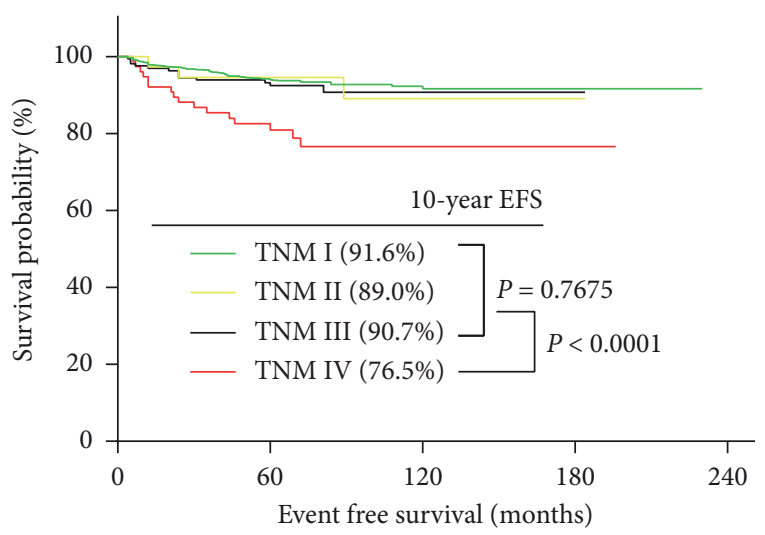

FIgURE 1: Kaplan-Meier survival curves for event-free survival (EFS) of PTC patients according to TNM stage in Zhejiang cohort. TNM staging in the figure was AJCC 7th edition based.

TABle 1: Demographics and clinicopathologic characteristics of patients with papillary thyroid cancer of TNM stages I-III $(N=1621)$.

\begin{tabular}{lcc}
\hline Demographic or characteristic & No. of patients & $\%$ \\
\hline Age at diagnosis, years & $44.1 \pm 11.8^{\mathrm{a}}$ & - \\
Sex & $44(8,89)^{\mathrm{b}}$ & - \\
$\quad$ Male & 384 & 23.7 \\
$\quad$ Female & 1237 & 76.3 \\
Bilaterality & & \\
$\quad$ Yes & 303 & 18.7 \\
$\quad$ No & 1318 & 81.3 \\
Tumor sizec (mm) & & \\
$\quad \leq 10$ & 988 & 60.9 \\
11-20 & 453 & 28.0 \\
$>20$ & 180 & 11.1 \\
Extrathyroidal extension & & \\
Yes & 108 & 6.7 \\
No & 1513 & 93.3 \\
Nodal status & & \\
N0/Nx & 1150 & 70.9 \\
N1a & 360 & 22.2 \\
N1b & 111 & 6.9 \\
TNM stage & & \\
I & 1420 & 87.6 \\
II & 37 & 2.3 \\
III & 164 & 10.1 \\
\hline
\end{tabular}

${ }^{\mathrm{a}}$ Mean \pm standard deviation; ${ }^{\mathrm{b}}$ median (range); ${ }^{\mathrm{c}}$ maximum tumor diameter.

recurrence and non-lymph node recurrence (including in situ relapse, contralateral lobe relapse, and distant metastasis). As shown in the supplementary Table 1, the rate of lymph node recurrence in younger PTC group (age $<30$ y) $(8.9 \%, 15 / 169)$ is significantly higher than that in group with age $\geq 30$ y $(3.5 \%, 51 / 1452, P<0.01)$. Similarly, the younger group also has higher incidence rate of non-lymph node recurrence $(6.0 \%, 10 / 169)$ when comparing with group with age $\geq 30 y(2.2 \%, 32 / 1452, P<0.01)$. These findings indicate that the elevated recurrence rate of younger patients was possibly due to all-cause recurrence rather than only lymph node recurrence.
3.4. Univariable and Multivariable Cox Regression Analyses. In the univariable Cox regression analysis, we found age at diagnosis, bilaterality, tumor size, extrathyroidal extension, and nodal status were related to EFS $(P<0.05)$ (Figure 3(a)). Sex was not found to be significant. While in multivariate Cox regression analysis, four independent prognostic factors for EFS were identified: age at diagnosis, bilaterality, tumor size, and nodal status. However, extrathyroidal extension was not independently correlated to recurrence $(P=0.693$; $\mathrm{HR}=1.13 ; 95 \% \mathrm{CI}=0.62-2.04$ ).

3.5. Prognostic Index (PI) and New Risk Stratification. Based on the parameters that we generated by multivariate Cox regression, PI values were calculated as follows: PI value $=0.597($ age at diagnosis $<30)+0.489(10<$ tumor size $\leq 20)+0.832 \quad(20<$ tumor size $)+0.483 \quad$ (bilateral $)+0.527$ (N1a) + 1.008 (N1b). Therefore, we applied the PI to divide PTC patients evenly into high-, median- and low-risk groups. The 10-year EFS rates for the high-, median-, and low-risk groups were well discriminated $(84.0 \%$ vs $92.0 \%$ vs $96.8 \%, P<0.0001$, Figure 3(b)).

3.6. Construction of the Nomogram and Validation. We constructed a nomogram to develop a new predictive tool for calculating probability of EFS at the individual level based on the factors identified as significant independent variables in the multivariate Cox regression analysis reported above. We randomly divided 1621 patients with TNM stages I-III into the modeling group $(n=1215)$ and the validation group $(n=406)$ at the rate of $3: 1$. There were no significant differences between the modeling group and validation group in all 6 clinicopathologic characteristics $(P>0.05$, Supplementary Table 2$)$. By integrating all significant independent factors for EFS, we constructed the nomogram based on the modeling data set (Figure 4). The discrimination (C-index) of our nomogram for EFS prediction was 0.70 ( $95 \% \mathrm{CI}, 0.64$ to 0.76 ), which was significantly higher $(P<0.0001)$ than the AJCC staging system (0.52). The calibration plot for the probability of EFS at 3, 5, or 10-year showed an optimal agreement between the prediction by our nomogram and actual observation (Supplementary Figures 2A-2C). In addition, in the external validation using the validation group, the $\mathrm{C}$-index was 0.65 (95\% CI, 0.55 to 0.75 ), and a calibration curve showed good agreement between prediction and observation in the probability of 5-year survival (Supplementary Figure 3).

\section{Discussion}

In this study, we found that the widely used TNM staging system is not efficient enough to predict tumor recurrence in PTC patients with TNM stages I, II and III. By calculating prognostic indexes based on a multivariate Cox model, we further constructed a new risk stratification system, in which the 10-year EFS rates for the low-, median-, and high-risk groups were well discriminated. Finally, we developed a new nomogram with high C-index to better estimate individualized prognosis for early-stage PTC patients. 


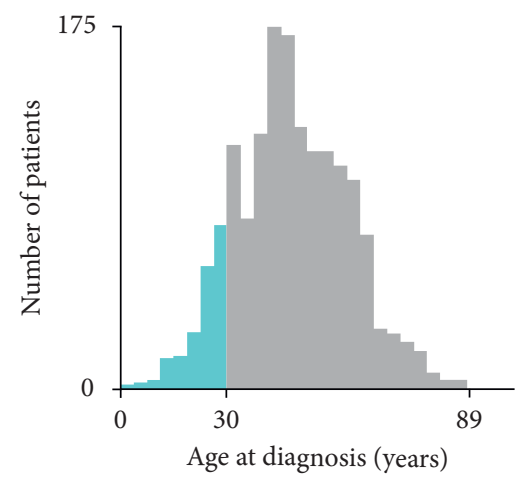

(a)

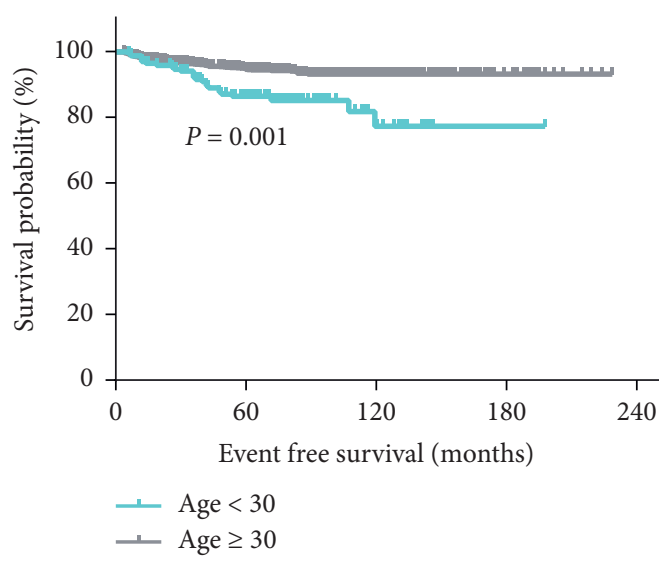

(b)

Figure 2: Analysis of age at diagnosis by X-tile software. (a) X-tile determined cutpoint of age and divided patients into high- and low-risk groups. (b) Kaplan-Meier survival curves for EFS according to age stratifications.

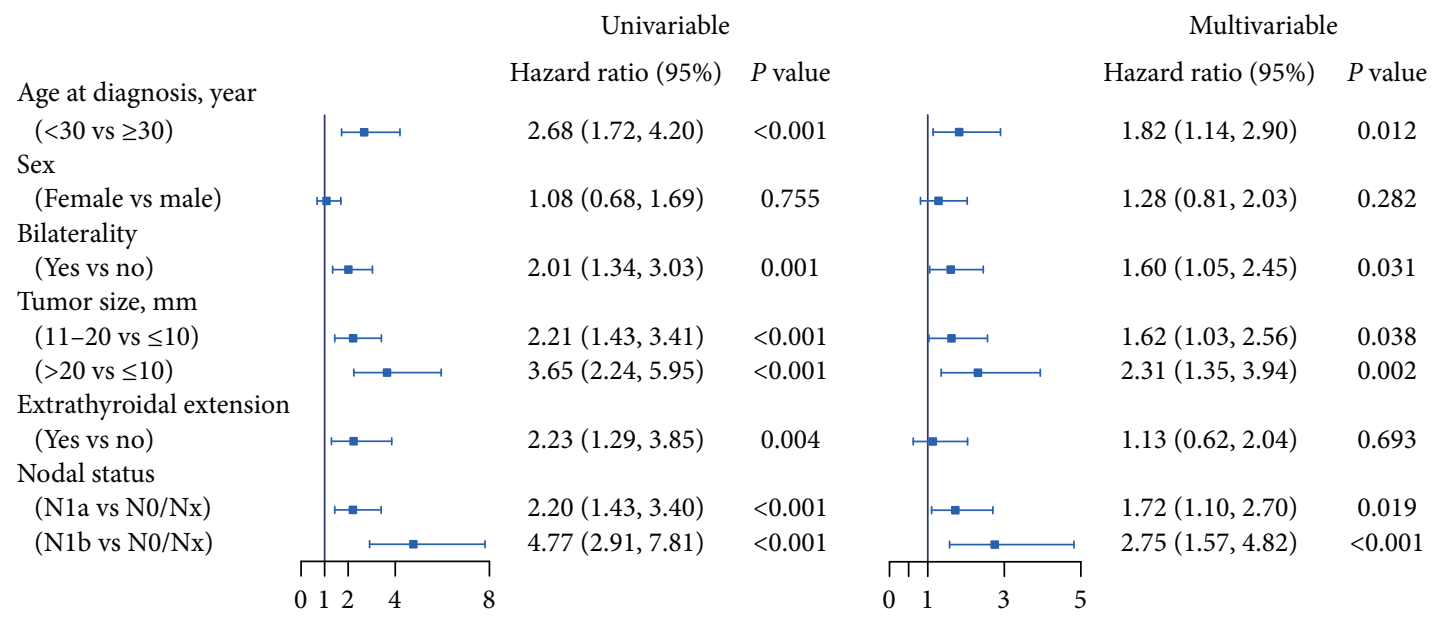

(a)

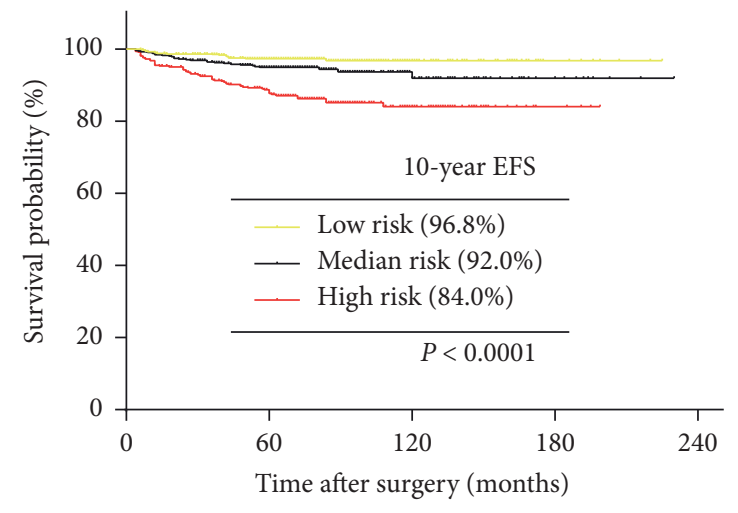

PI value $=0.597($ age at diagnosis $<30)+0.489$ $(10<$ tumor size $\leq 20)+0.832$ (tumor size $>20)$ $+0.483($ bilateral $)+0.527(\mathrm{~N} 1 \mathrm{a})+1.008(\mathrm{~N} 1 \mathrm{~b})$

Low risk $(\mathrm{PI}=0, N=653)$

Median risk $(0<\mathrm{PI} \leq 0.832, N=505)$

High risk $(\mathrm{PI}>0.832, N=463)$

(b)

FIGURE 3: Cox regression analysis and risk stratification for papillary thyroid cancer. (a) Univariate and multivariate cox regression analysis of EFS for papillary thyroid cancer. (b) Kaplan-Meier survival curves for disease-free survival according to risk stratification. The PI value computational formula was based on multivariate Cox regression analysis.

There exists a long-lasting debate on whether and how the age influences the prognosis of thyroid cancer. Orosco et al. analyzed 85,740 patients in the SEER database and found no single year between $25-55$ can be chosen to yield a high hazard ratio for survival, which suggests none of them should be served as a unique cutoff [16]. Since the majority of 


Points
Age (years)

FIGURE 4: The nomogram for predicting event-free survival (EFS) of papillary thyroid cancer (the nomogram was used as follows: possible values of each variable from an individual patient are located on each variable axis, and a line is drawn upward to determine the points got for each variable on the point axis. The sum of these points is located on the total point axis. Then, a line is drawn downward to the survival axes to determine the likelihood of 3-, 5-, or 10-year EFS. A specific example is shown in Supplementary Figure 1).

PTCs have good prognosis with high survival rate of 10 and even 20 years, the factors that affect recurrence should be better delineated. Our data identified the age of 30 as the optimal cutoff for recurrence and was further confirmed as an independent risk factor by the multivariable Cox regression analysis. Similarly, Cho et al found the recurrence rates of PTC were higher in patients under 35, among which $15.7 \%$ of recurrences were under the age less than 25 years [17]. Here, we found that PTC patients of young age had higher potential risk of recurrence. For this group of patients, we suggest increasing the intensity and frequency of follow-up.

Bilateral PTC has been traditionally discovered after total thyroidectomy with the incidence varying from $20 \%$ to $60 \%$ according to the literature [18]. However, few data specifically examining the prognostic implications of bilaterality in PTC have been reported. Pellegriti et al. first evaluated the association between bilaterality and recurrence risk in 299 PTCs [19]. Subsequently, we detailed this relationship in 891 PTC patients [20] and further confirmed in 2,211 patients in the later follow-up study [13]. We found that bilateral PTC is common, occurring in $19.9 \%$ of our cohort, and that bilaterality is associated with poorer prognosis. Our data presented here are the first to identify bilaterality as an independent risk factor for recurrence in PTC, supporting total thyroidectomy as the preferred surgical approach in patients with bilateral disease. In a recent study of 3282 patients with micro-PTC, Hwangbo et al. also found bilaterality as an independent risk factor for longterm recurrence [21]. The clonality study from our group revealed most bilateral PTC tumors are of similar clonal origin, suggesting this specific disease is a consequence of a single primary with subsequent intrathyroidal metastasis to the contralateral thyroid lobe. This observation provides a molecular rationale as to why the outcome of bilateral PTCs was observed to be poorer in this dataset [22].

The AJCC staging system has been criticized for its inability to accurately predict the prognosis of PTC patients, especially for the low risk majority $[23,24]$. While the incidence of PTC has increased dramatically in recent decades, the mortality has remained relatively unchanged, implying that most of the increase is due to low-risk PTCs $[2,5,25]$. Distinguishing the aggressive low-risk tumors from the indolent majority could help to avoid overtreatment of the low-risk PTCs. Various risk stratification systems have been proposed, such as the MACIS (metastasis-age-completeness of resection-invasion-size) prognostic scoring system [26], and the ATA active surveillance system [7]. Nomograms also been applied to predict cancer-specific survival or recurrence of thyroid cancer $[11,27]$. In addition, studies have reported that nomograms resulted in more accurate prognostic prediction than the traditional staging systems for patients with colon, stomach, and liver cancer [28-30]. In the current study, we developed a prognostic nomogram for predicting recurrence risk in PTC patients with TNM stages I-III. To our knowledge, this is the first risk stratification system that specifically evaluates recurrence in PTC patients with TNM stages I-III. When compared with conventional TNM staging system, the nomogram showed better predictive accuracy for recurrence, and external validation showed that the model had good stability.

The limitation of our study is that it was a retrospective, single-institution research. Due to the higher proportion of microcarcinoma in our study, the conclusions of this study 
are limited and need to be strictly verified in groups with relatively low proportion of microcarcinoma, such as Europe and the United States population. And, the TNM data were based on the 7 th edition of the AJCC staging system. The newly published 8th edition possesses some changes including the increased age cutoff from 45 to $55 \mathrm{y}$ and the removal of the minimal extrathyroidal extension from the definition of T3 [31]. However, AJCC staging was mainly developed to predict risk for death and the benefits of 8th edition in predicting recurrence which we focused in this study remain unknown $[32,33]$. We plan to further compare the difference of EFS between these two staging systems in our later study. Another limitation is the lack of some other important variables that could be informative, such as information on RAI treatment and the genetic alteration status (BRAF, RAS, TERT etc). We plan to validate the new risk stratification system by some cohorts outside and to further combine genetic alterations like BRAF and TERT promoter mutations in that system.

\section{Conclusion}

In conclusion, this study provides initial evidence that the EFS of early-stage PTC patients (TNM stages I, II, and III) cannot be well discriminated. By using univariate and multivariate Cox regression analysis, we established a new risk stratification system for this specific group of PTCs.

\section{Data Availability}

The data used to support the findings of this study are included within the article.

\section{Conflicts of Interest}

The authors declare that they have no conflicts of interest.

\section{Authors' Contributions}

Yongfeng Ding and Zhuochao Mao contributed equally to this work.

\section{Acknowledgments}

This study was supported by the grants from the National Natural Science Foundation of China (nos. 81772853 and 81972495), National Natural Science Foundation of Zhejiang (nos. LY18H160041 and LQ18H120002), Key Project of Scientific and Technological Innovation of Zhejiang Province (no. 2015C03031), and Key Project of Scientific and Technological Innovation of Hangzhou (no. 20131813A08).

\section{Supplementary Materials}

Supplementary Table 1: comparison of the rate of lymph node recurrence and non-lymph node recurrence in different age groups. Supplementary Table 2: comparison demographics and clinicopathologic characteristics of patients with papillary thyroid carcinoma. Supplementary Figure 1: an example for the nomogram model; a 50-year-old female with bilateral PTC, whose tumor size is between 10 and $20 \mathrm{~mm}$ and nodal status is N1b. Her total score in this nomogram is about 17.58, and 3-, 5- and 10-year EFSs are about $86 \%, 80 \%$, and $76 \%$, specifically. Supplementary Figure 2: the calibration curve for predicting patient EFS survival at (A) 3 years, (B) 5 years, and (C) 10 years in the modeling cohort. $X$-axis, nomogram-predicted probability of EFS; $Y$-axis, actual EFS. Supplementary Figure 3: the calibration curve for predicting patient survival at 5 years in the validation cohort. (Supplementary Materials)

\section{References}

[1] C. La Vecchia, M. Malvezzi, C. Bosetti et al., "Thyroid cancer mortality and incidence: a global overview," International Journal of Cancer, vol. 136, no. 9, pp. 2187-2195, 2015.

[2] H. S. Ahn, H. J. Kim, and H. G. Welch, "Korea's thyroidcancer "epidemic"-screening and overdiagnosis," New England Journal of Medicine, vol. 371, no. 19, pp. 1765-1767, 2014.

[3] W. Chen, R. Zheng, P. D. Baade et al., "Cancer statistics in China, 2015," CA: A Cancer Journal for Clinicians, vol. 66, no. 2, pp. 115-132, 2016.

[4] J. A. Fagin and S. A. Wells Jr., "Biologic and clinical perspectives on thyroid cancer," New England Journal of Medicine, vol. 375, no. 11, pp. 1054-1067, 2016.

[5] L. Davies and H. G. Welch, "Current thyroid cancer trends in the United States," JAMA Otolaryngology-Head and Neck Surgery, vol. 140, no. 4, pp. 317-322, 2014.

[6] R. M. Tuttle, R. I. Haddad, D. W. Ball et al., "Thyroid carcinoma, version 2.2014," Journal of the National Comprehensive Cancer Network, vol. 12, no. 12, pp. 1671-1680, 2014.

[7] B. R. Haugen, E. K. Alexander, K. C. Bible et al., "2015 American thyroid association management guidelines for adult patients with thyroid nodules and differentiated thyroid cancer: the American thyroid association guidelines task force on thyroid nodules and differentiated thyroid cancer, thyroid," Thyroid, vol. 26, no. 1, pp. 1-133, 2016.

[8] S. Frangos, I. P. Iakovou, R. J. Marlowe et al., "Acknowledging gray areas: 2015 vs. 2009 American thyroid association differentiated thyroid cancer guidelines on ablating putatively low-intermediate-risk patients," European Journal of Nuclear Medicine and Molecular Imaging, vol. 44, no. 2, pp. 185-189, 2017.

[9] D. S. Cooper, G. M. Doherty, B. R. Haugen et al., "Revised American thyroid association management guidelines for patients with thyroid nodules and differentiated thyroid cancer," Thyroid, vol. 19, no. 11, pp. 1167-1214, 2009.

[10] A. Iasonos, D. Schrag, G. V. Raj, and K. S. Panageas, "How to build and interpret a nomogram for cancer prognosis," Journal of Clinical Oncology, vol. 26, no. 8, pp. 1364-1370, 2008.

[11] K. A. Pathak, A. Mazurat, P. Lambert, T. Klonisch, R. W., and Nason, "Prognostic nomograms to predict oncological outcome of thyroid cancers," The Journal of Clinical Endocrinology and Metabolism, vol. 98, no. 12, pp. 4768-4775, 2013.

[12] L. Yang, W. Shen, and N. Sakamoto, "Population-based study evaluating and predicting the probability of death resulting from thyroid cancer and other causes among patients with thyroid cancer," Journal of Clinical Oncology, vol. 31, no. 4, pp. 468-474, 2013.

[13] W. Wang, X. Su, K. He et al., "Comparison of the clinicopathologic features and prognosis of bilateral versus unilateral 
multifocal papillary thyroid cancer: an updated study with more than 2000 consecutive patients," Cancer, vol. 122, no. 2 , pp. 198-206, 2016.

[14] R. L. Camp, M. Dolled-Filhart, and D. L. Rimm, "X-tile: a new bio-informatics tool for biomarker assessment and outcomebased cut-point optimization," Clinical Cancer Research, vol. 10, no. 21, pp. 7252-7259, 2004.

[15] F. E. Harrell Jr., "RMS: regression modeling strategies, R package version 5.1-0," 2019, http://CRAN.Rproject.org/ package $=$ rms.

[16] R. K. Orosco, T. Hussain, K. T. Brumund, D. K. Oh, D. C. Chang, and M. Bouvet, "Analysis of age and disease status as predictors of thyroid cancer-specific mortality using the surveillance, epidemiology, and endresults database," Thyroid, vol. 25, no. 1, pp. 125-132, 2015.

[17] J. S. Cho, J. H. Yoon, M. H. Park et al., "Age and prognosis of papillary thyroid carcinoma: retrospective stratification into three groups," Journal of the Korean Surgical Society, vol. 83, no. 5, pp. 259-266, 2012.

[18] R. Elisei, E. Molinaro, L. Agate et al., "Are the clinical and pathological features of differentiated thyroid carcinoma really changed over the last 35 years? Study on 4187 patients from a single Italian institution to answer this question," The Journal of Clinical Endocrinology and Metabolism, vol. 95, no. 4, pp. 1516-1527, 2010.

[19] G. Pellegriti, C. Scollo, G. Lumera, C. Regalbuto, R. Vigneri, and A. Belfiore, "Clinical behavior and outcome of papillary thyroid cancers smaller than $1.5 \mathrm{~cm}$ in diameter: study of 299 cases," The Journal of Clinical Endocrinology and Metabolism, vol. 89, no. 8, pp. 3713-3720, 2004.

[20] W. Wang, W. Zhao, H. Wang et al., "Poorer prognosis and higher prevalence of BRAF V600E mutation in synchronous bilateral papillary thyroid carcinoma," Annals of Surgical Oncology, vol. 19, no. 1, pp. 31-36, 2012.

[21] Y. Hwangbo, J. M. Kim, Y. J. Park et al., "Long-term recurrence of small papillary thyroid cancer and its risk factors in a Korean multicenter study," The Journal of Clinical Endocrinology and Metabolism, vol. 102, no. 2, pp. 625-633, 2017.

[22] W. Wang, H. Wang, X. Teng et al., "Clonal analysis of bilateral, recurrent, and metastatic papillary thyroid carcinomas," Human Pathology, vol. 41, no. 9, pp. 1299-1309, 2010.

[23] B. Cady and R. Rossi, "An expanded view of risk-group definition in differentiated thyroid carcinoma," Surgery, vol. 104, no. 6, pp. 947-953, 1988.

[24] Q. Cheng, X. Li, C. R. Acharya, T. Hyslop, and J. A. Sosa, “A novel integrative risk index of papillary thyroid cancer progression combining genomic alterations and clinical factors," Oncotarget, vol. 8, no. 10, pp. 16690-16703, 2017.

[25] L. Davies and H. G. Welch, "Increasing incidence of thyroid cancer in the United States, 1973-2002," JAMA, vol. 295, no. 18, pp. 2164-2167, 2006.

[26] I. D. Hay, E. J. Bergstralh, J. R. Goellner, J. R. Ebersold, and C. S. Grant, "Predicting outcome in papillary thyroid carcinoma: development of a reliable prognostic scoring system in a cohort of 1779 patients surgically treated at one institution during 1940 through 1989," Surgery, vol. 114, no. 6, pp. 1050-1057, 1993.

[27] B. H. H. Lang, C. K. H. Wong, H. W. Yu, and K. E. Lee, "Postoperative nomogram for predicting disease-specific death and recurrence in papillary thyroid carcinoma," Head and Neck, vol. 38, no. S1, pp. E1256-E1263, 2016.

[28] M. R. Weiser, R. G. Landmann, M. W. Kattan et al., "Individualized prediction of colon cancer recurrence using a nomogram," Journal of Clinical Oncology, vol. 26, no. 3, pp. $380-385,2008$.

[29] D.-S. Han, Y.-S. Suh, S.-H. Kong et al., "Nomogram predicting long-term survival after D2 gastrectomy for gastric cancer," Journal of Clinical Oncology, vol. 30, no. 31, pp. 3834-3840, 2012.

[30] Y. Wang, J. Li, Y. Xia et al., "Prognostic nomogram for intrahepatic cholangiocarcinoma after partial hepatectomy," Journal of Clinical Oncology, vol. 31, no. 9, pp. 1188-1195, 2013.

[31] E. S. Amin, F. L. Greene, S. B. Edge et al., AJCC Cancer Staging Manual, Springer International Publishing, New York, NY, USA, 8th edition, 2017.

[32] N. D. Perrier, J. D. Brierley, and R. M. Tuttle, "Differentiated and anaplastic thyroid carcinoma: major changes in the American joint committee on cancer eighth edition cancer staging manual," CA: A Cancer Journal for Clinicians, vol. 68, no. 1, pp. 55-63, 2018.

[33] R. M. Tuttle, B. Haugen, and N. D. Perrier, "Updated American joint committee on cancer/tumor-node-metastasis staging system for differentiated and anaplastic thyroid cancer (eighth edition): what changed and why?," Thyroid, vol. 27, no. 6, pp. 751-756, 2017. 


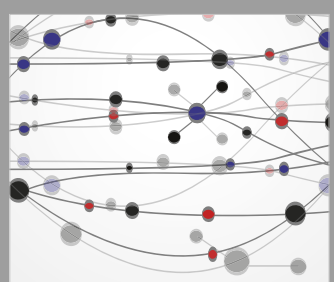

The Scientific World Journal
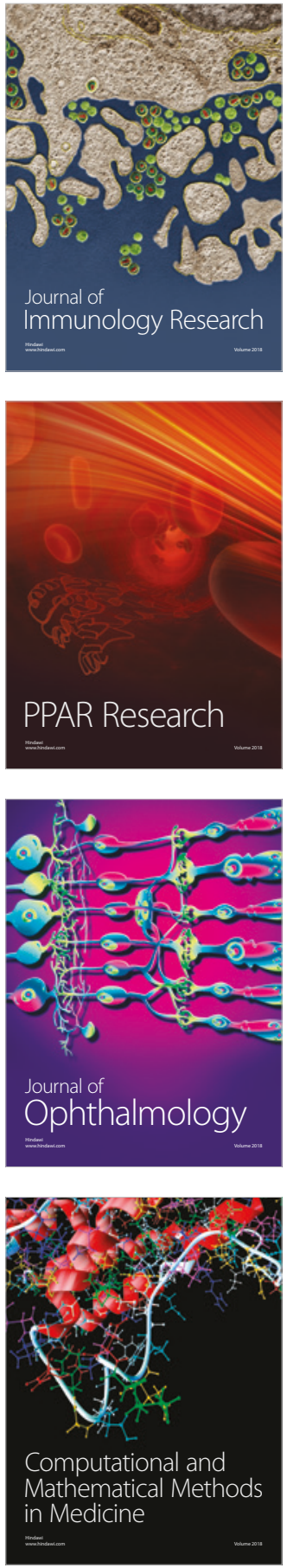

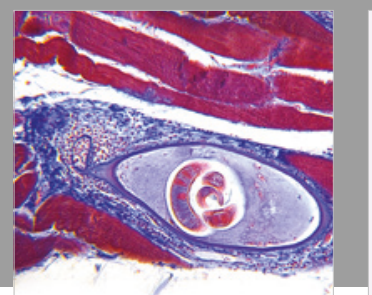

Gastroenterology Research and Practice

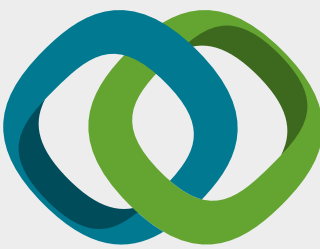

\section{Hindawi}

Submit your manuscripts at

www.hindawi.com
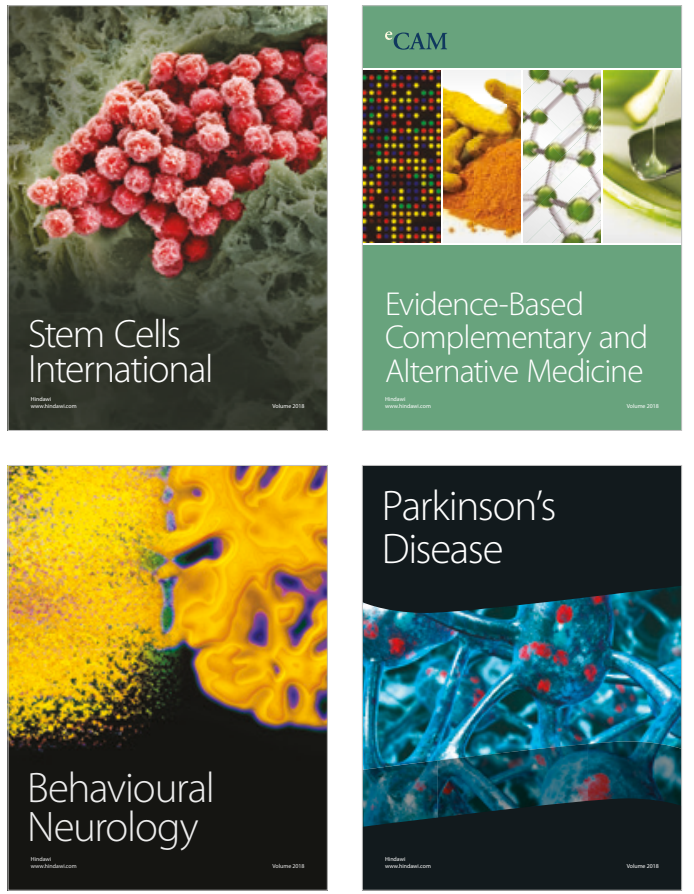

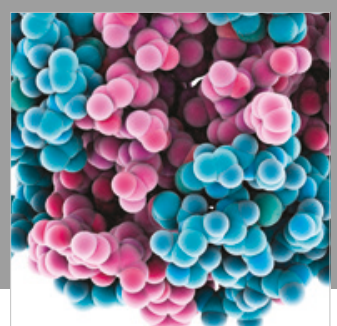

ournal of

Diabetes Research

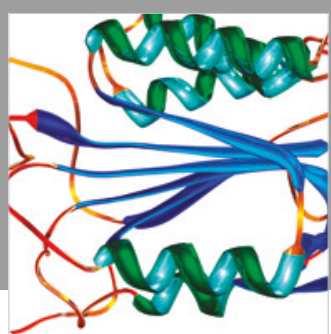

Disease Markers
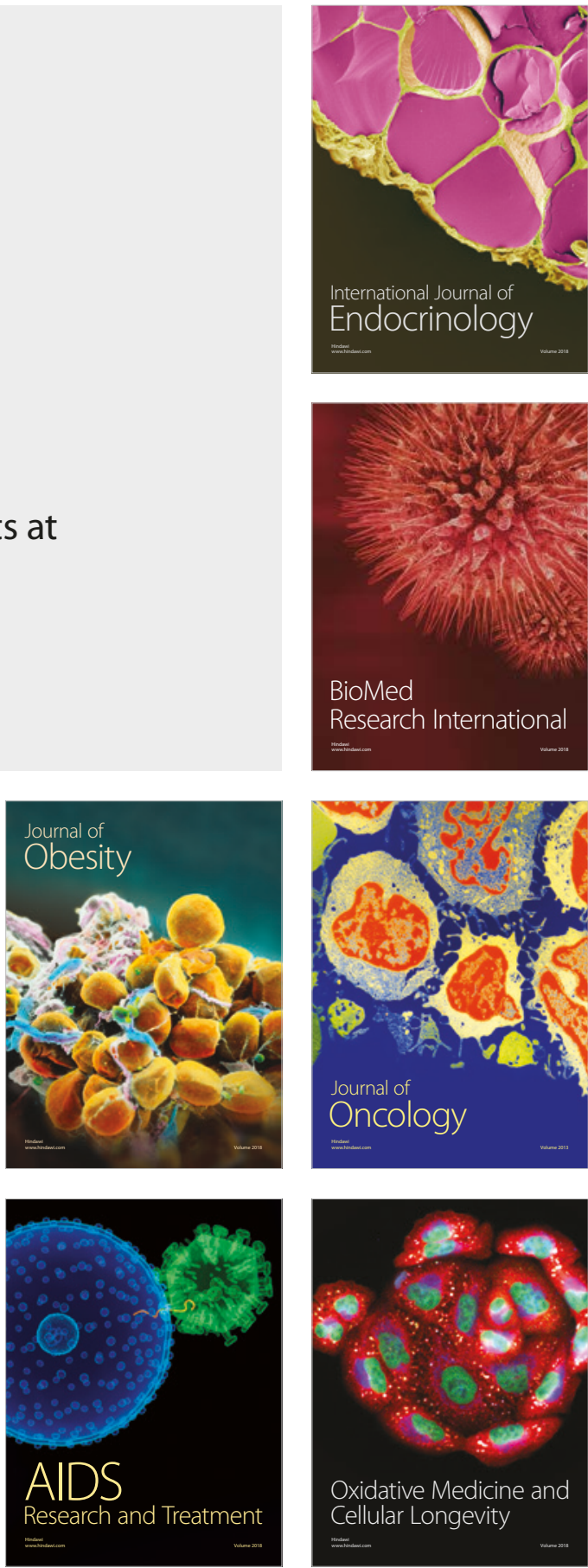